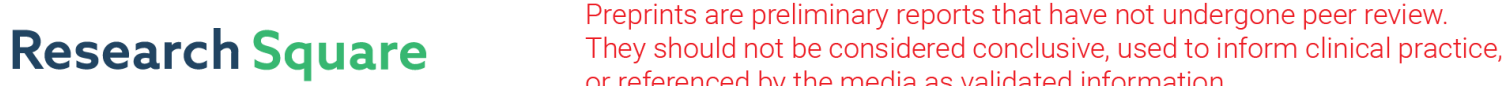 or referenced by the media as validated information. \\ Lookback Option Pricing Problem of Uncertain Mean-reverting Currrency Model
}

\section{Yang Liu ( $\sim 2016$ liuyang@tongji.edu.cn )}

Liaocheng University

\section{Liying Liu}

Liaocheng University

\section{Research Article}

Keywords: uncertainty theory, uncertain differential equation, lookback option pricing, currency model

Posted Date: August 10th, 2021

DOl: https://doi.org/10.21203/rs.3.rs-783868/v1

License: (c) (i) This work is licensed under a Creative Commons Attribution 4.0 International License. Read Full License 


\title{
Lookback option pricing problem of uncertain mean-reverting currency model
}

\author{
Yang $\operatorname{Liu}^{a^{*}}$, Liying $\operatorname{Liu}^{b}$ \\ $a^{*}$. School of Business, Liaocheng University, Liaocheng 252059, China \\ b. School of Mathematical Sciences, Liaocheng University, Liaocheng 252059, China \\ 2016liuyang@tongji.edu.cn, lyliu001@163.com
}

\begin{abstract}
A lookback option is a maturity option that pays off based on the maximum or minimum stock price over the life of the option. This paper investigates the problem of pricing a lookback option based on the uncertain mean-reverting currency model and designs the algorithms to calculate the formulations. Furthermore, discussions about parameters and results are drawn in the paper.
\end{abstract}

Keywords: uncertainty theory, uncertain differential equation, lookback option pricing, currency model

\section{Introduction}

The option pricing problem which was proposed by Bachelier [1] in 1900 was constantly popular. But it has not been solved scientifically until stochastic process differential equation was founded in 1951 by Ito [2]. Following that, Black and Scholes [3] investigated the famous Black-Scholes option pricing formula in 1973, which assumed the stock price followed a geometric Wiener process. Considering the jump phenomena of stock price, Merton [4] reconstructed the model. After that, amount of stochastic differential equations had been used to describe the stock price and various option pricing formulas were derived.

In the application of Black-Scholes option pricing formula, investors pointed out the strict restriction of the prerequisite to use the formula. In addition, two paradoxes were founded by Liu [5] show that the stock price in real financial market is impossible to follow stochastic differential equations.

To deal with the conflicts between real mark and probability theory, uncertainty theory was built by Liu [6] in 2007, which was based on normality, duality, subadditivity and product axioms. Then it was applied to amount of fields, such as uncertain programming [7], uncertain logic [8], uncertain reliability analysis and uncertain risk analysis [9]. The basic concept uncertain variable in uncertainty theory was defined by Liu [6] to model the quantity associated with human uncertainty. Meanwhile, uncertainty distribution was given to describe uncertain variables, excepted value was provided to rank uncertain variables.

Uncertain process, a sequence of uncertain variables driven by time, was explored by Liu [10] in 2008 to model the evolution of uncertain phenomena. As a special and important type of uncertain process, Liu [7] designed Liu process within the framework of uncertainty theory as a counterpart of Wiener process. Following that, Liu [11] began the pioneer work of uncertain finance based on the assumption of the stock price's fluctuation follows a geometric Liu process. After that, its European option, American option [12] and Asian option [13] [14] pricing formulas were derived respectively.

Lookback option is a path-dependent option that offers the payoff depends on the maximum or minimum 
value of the underlying asset price. The pricing problem of lookback option has been widely discussed since it was first valuated by Goldman et al. [15]. Conze and Viswannathan [16] used the joint distribution of the spot price and its historical maximum (or minimum) to obtain the closed-form valuation formulas. Quanto lookback option price formulas were derived by Dai et al. [17]. With framework of uncertainty theory, Zhang et al. [18] firstly investigated the lookback option problem. Based on that, Gao et al. [19] developed the lookback call and put option pricing formulas following the uncertain exponential Ornstein-Uhlenbeck model.

By viewing the foreign exchange rate as uncertain process and describing an uncertain differential equation as Liu process, an uncertain currency model was proposed by Liu et al. [20] in 2015. Based on the foundation, Shen and Yao [21] presented a mean-reverting currency model by considering the exchange rate in the long term in the real global market. Sheng [22] investigated the Asian currency option pricing problem following the mean-reverting stock model. Then Wang and Ning [23] built a uncertain currency model with floating interest rates by considering the uncertain fluctuations in the financial market from time to time.

The existing scholarship in this field, however, tended to overlook the combination of lookback option and uncertain mean-reverting currency model. Following the previous work on option pricing problem within framework of uncertainty theory lead, this paper will combine lookback option and uncertain meanreverting currency model to derive the lookback option pricing formulas for the uncertain mean-reverting currency model. The rest of the paper is organized as follows. In next section, this paper will review some preliminary knowledge in uncertainty theory. In Section 3, the lookback call option will be introduced and its pricing formula will be derived. An algorithm will be designed to calculate the price numerically, a numerical experiment will be given and the sensibility of single variable will be analysed finally. In Section 4 , the lookback put option will be discussed in the similar way.In Section 5, the conclusion will give some conclusions.

\section{Preliminaries}

In this section, some preliminary concepts from uncertainty theory as needed are reviewed for further understanding the paper.

\subsection{Uncertain variable}

Definition 1. (Liu [6]) Let $\Gamma$ be a nonempty set, and $\mathcal{L}$ be a $\sigma$-algebra over $\Gamma$. A set function $\mathcal{M}: \mathcal{L} \rightarrow[0,1]$ is called an uncertain measure if it satisfies the following axioms:

Axiom 1. (Normality Axiom) $\mathcal{M}\{\Gamma\}=1$ for the universal set $\Gamma$.

Axiom 2. (Duality Axiom) $\mathcal{M}\{\Lambda\}+\mathcal{M}\left\{\Lambda^{c}\right\}=1$ for any event $\Lambda$.

Axiom 3. (Subadditivity Axiom) For every countable sequence of events $\Lambda_{1}, \Lambda_{2}, \cdots$, we have

$$
\mathcal{M}\left\{\bigcup_{i=1}^{\infty} \Lambda_{i}\right\} \leq \sum_{i=1}^{\infty} \mathcal{M}\left\{\Lambda_{i}\right\} .
$$

Note that the triplet $(\Gamma, \mathcal{L}, \mathcal{M})$ is called an uncertainty space.

Besides, the product uncertain measure on the product $\sigma$-algebra was defined by Liu [11] as follows.

Axiom 4. (Product Axiom) Let $\left(\Gamma_{k}, \mathcal{L}_{k}, \mathcal{M}_{k}\right)$ be uncertainty spaces for $k=1,2, \cdots, n$. The product uncertain measure $\mathcal{M}$ is an uncertain measure satisfying

$$
\mathcal{M}\left\{\prod_{k=1}^{\infty} \Lambda_{k}\right\}=\bigwedge_{k=1}^{\infty} \mathcal{M}_{k}\left\{\Lambda_{k}\right\}
$$


where $\Lambda_{k}$ are arbitrarily chosen events from $\mathcal{L}_{k}$ for $k=1,2, \cdots, n$, respectively.

Definition 2. (Liu [6]) An uncertain variable $\xi$ is a measurable function from an uncertainty space $(\Gamma, \mathcal{L}, \mathcal{M})$ to the set of real numbers, i.e., for any Borel set $B$, the set $\{\gamma \in \Gamma \mid \xi(\gamma) \in B\}$ is an event in $\mathcal{L}$.

Definition 3. (Liu [6]) The uncertainty distribution $\Phi$ of an uncertain variable $\xi$ is defined by

$$
\Phi(x)=\mathcal{M}\{\xi \leq x\}
$$

for any real number $x$.

Liu and Lio[24] proposed that A real-valued function $\Phi(x)$ on $\Re$ is an uncertainty distribution if and only if it is a monotone increasing function satisfying

$$
0 \leq \Phi(x) \leq 1, \quad \Phi(x) \not \equiv 0, \quad \Phi(x) \not \equiv 1, \quad \Phi\left(x_{0}\right)=1
$$

if $\Phi(x)=1$ for any $x>x_{0}$.

Definition 4. (Liu [25]) Let $\xi$ be an uncertain variable with regular uncertainty distribution $\Phi(x)$. Then the inverse function $\Phi^{-1}(\alpha)$ is called the inverse uncertainty distribution of $\xi$.

Example 1. An uncertain variable $\xi$ is called linear if it has a linear uncertainty distribution

$$
\Phi(x)=\left\{\begin{array}{cl}
0, & \text { if } x<a \\
(x-a) /(b-a), & \text { if } a \leq x<b \\
1, & \text { if } x \geq b
\end{array}\right.
$$

denoted by $\mathcal{L}(a, b)$, where $a$ and $b$ are real numbers with $a<b$. The inverse uncertainty distribution of linear uncertain variable $\mathcal{L}(a, b)$ is

$$
\Phi^{-1}(\alpha)=(1-\alpha) a+\alpha b
$$

Example 2. An uncertain variable $\xi$ is called normal if it has a normal uncertainty distribution

$$
\Phi(x)=\left(1+\exp \left(\frac{\pi(e-x)}{\sqrt{3} \sigma}\right)\right)^{-1}, \quad x \in \Re
$$

denoted by $\mathcal{N}(e, \sigma)$, where $e$ and $\sigma$ are real numbers with $\sigma>0$. The inverse uncertainty distribution of normal uncertain variable $\mathcal{N}(e, \sigma)$ is

$$
\Phi^{-1}(\alpha)=e+\frac{\sigma \sqrt{3}}{\pi} \ln \frac{\alpha}{1-\alpha}
$$

Definition 5. (Liu [6]) Let $\xi$ be an uncertain variable. Then the expected value $\mathrm{E}[\xi]$ is

$$
\mathrm{E}[\xi]=\int_{0}^{+\infty} \mathcal{M}\{\xi \geq x\} d x-\int_{-\infty}^{0} \mathcal{M}\{\xi \leq x\} d x
$$

provided that at least one of the two integrals is finite.

Theorem 2.1. (Liu [6]) Let $\xi$ be an uncertain variable with uncertainty distribution $\Phi$. Then

$$
\mathrm{E}[\xi]=\int_{0}^{+\infty}(1-\Phi(x)) d x-\int_{-\infty}^{0} \Phi(x) d x .
$$




\subsection{Uncertain differential equaiton}

An uncertain process is a sequence of uncertain variables indexed by time. A formal definition of uncertain process is stated as follows.

Definition 6. (Liu [10]) Let $T$ be a totally ordered set (e.g. time) and let $(\Gamma, \mathcal{L}, \mathcal{M})$ be an uncertainty space. An uncertain process is a function $X_{t}(\gamma)$ from $T \times(\Gamma, \mathcal{L}, \mathcal{M})$ to the set of real numbers such that $\left\{X_{t} \in B\right\}$ is an event for any Borel set $B$ of real numbers at each time $t$.

Definition 7. (Liu [26]) Uncertain processes $X_{1 t}, X_{2 t}, \cdots, X_{n t}$ are said to be independent if for any positive integer $k$ and any times $t_{1}, t_{2}, \cdots, t_{k}$, the uncertain vectors

$$
\xi_{i}=\left(X_{i t_{1}}, X_{i t_{2}}, \cdots, X_{i t_{k}}\right), \quad i=1,2, \cdots, n
$$

are independent, i.e., for any Borel sets $B_{1}, B_{2}, \cdots, B_{n}$ of $k$-dimensional real vectors, we have

$$
\mathcal{M}\left\{\bigcap_{i=1}^{n}\left(\xi_{i} \in B_{i}\right)\right\}=\bigwedge_{i=1}^{n} \mathcal{M}\left\{\xi_{i} \in B_{i}\right\} .
$$

An uncertain process $X_{t}$ is said to have independent increments if

$$
X_{t_{0}}, X_{t_{1}}-X_{t_{0}}, X_{t_{2}}-X_{t_{1}}, \cdots, X_{t_{k}}-X_{t_{k-1}}
$$

are independent uncertain variables where $t_{0}$ is the initial time and $t_{1}, t_{2}, \cdots, t_{k}$ are any times with $t_{0}<$ $t_{1}<\cdots<t_{k}$. An uncertain process $X_{t}$ is said to have stationary increments if, for any given $t>0$, the increments $X_{s+t}-X_{s}$ are identically distributed uncertain variables for all $s>0$.

Definition 8. (Liu [11]) An uncertain process $C_{t}$ is said to be a Liu process if

(i) $C_{0}=0$ and almost all sample paths are Lipschitz continuous,

(ii) $C_{t}$ has stationary and independent increments,

(iii) every increment $C_{t+s}-C_{s}$ is a normal uncertain variable with an uncertainty distribution

$$
\Phi_{t}(x)=\left(1+\exp \left(-\frac{\pi x}{\sqrt{3} t}\right)\right)^{-1}, \quad x \in \Re .
$$

Definition 9. (Liu [11]) Let $X_{t}$ be an uncertain process and let $C_{t}$ be a Liu process. For any partition of closed interval $[a, b]$ with $a=t_{1}<t_{2}<\cdots<t_{k+1}=b$, the mesh is written as

$$
\Delta=\max _{1 \leq i \leq k}\left|t_{i+1}-t_{i}\right| .
$$

Then Liu integral of $X_{t}$ with respect to $C_{t}$ is

$$
\int_{a}^{b} X_{t} \mathrm{~d} C_{t}=\lim _{\Delta \rightarrow 0} \sum_{i=1}^{k} X_{t_{i}} \cdot\left(C_{t_{i+1}}-C_{t_{i}}\right),
$$

provided that the limit exists almost surely and is finite. In this case, the uncertain process $X_{t}$ is said to be integrable.

Theorem 2.2. (Yao-Chen [27]) Let $Z_{t}$ be an independent sample-continuous increment process. Then $Z_{t}$ has a regular uncertainty distribution $\Phi_{t}(x)$. Let $J(x)$ be a real value strictly increasing function. It shows that the time integral

$$
Z_{T}=\int_{0}^{T} J\left(Z_{t}\right) d t
$$

has an inverse uncertainty distribution

$$
\Psi_{T}^{-1}(\alpha)=\int_{0}^{T} J\left(\Psi_{t}^{-1}(\alpha)\right) d t .
$$


Definition 10. (Liu [10]) Let $C_{t}$ be a Liu process, assume $f$ and $g$ are continuous functions. Then

$$
d X_{t}=f\left(t, X_{t}\right) d t+g\left(t, X_{t}\right) d C_{t}
$$

is called an uncertain differential equation. A solution is an uncertain process $X_{t}$ that satisfies the equation identically in $t$.

Definition 11. (Yao-Chen[27]) The $\alpha$-path $(0<\alpha<1)$ of an uncertain differential equation

$$
d X_{t}=f\left(t, X_{t}\right) d t+g\left(t, X_{t}\right) d C_{t}
$$

with an initial value $X_{0}$ is a deterministic function $X_{t}^{\alpha}$ with respect to that solves the corresponding ordinary differential equation

$$
d X_{t}^{\alpha}=f\left(t, X_{t}^{\alpha}\right) d t+\left|g\left(t, X_{t}^{\alpha}\right)\right| \Phi^{-1}(\alpha) d t .
$$

where $\Phi^{-1}(\alpha)$ is the inverse uncertainty distribution of standard normal uncertain variable,i.e.,

$$
\Phi^{-1}(\alpha)=\frac{\sqrt{3}}{\pi} \ln \frac{\alpha}{1-\alpha}, 0<\alpha<1
$$

Theorem 2.3. (Yao-Chen [27]) Let $X_{t}$ and $X_{t}^{\alpha}$ be the solution and $\alpha$-path of the uncertain differential equation

$$
d X_{t}=f\left(t, X_{t}\right) d t+g\left(t, X_{t}\right) d C_{t},
$$

respectively. Then,

$$
\begin{gathered}
\mathcal{M}\left\{X_{t} \leq X_{t}^{\alpha}, \forall t\right\}=\alpha, \\
\mathcal{M}\left\{X_{t}>X_{t}^{\alpha}, \forall t\right\}=1-\alpha .
\end{gathered}
$$

This formula is well known as Yao-Chen formula.

Theorem 2.4. (Yao-Chen [27]) Let $X_{t}$ and $X_{t}^{\alpha}$ be the solution and $\alpha$-path of the uncertain differential equation

$$
d X_{t}=f\left(t, X_{t}\right) d t+g\left(t, X_{t}\right) d C_{t},
$$

has an inverse uncertainty distribution

$$
\Psi_{t}^{-1}(\alpha)=X_{t}^{\alpha}
$$

Theorem 2.5. (Yao[28]) Let $X_{t}$ and $X_{t}^{\alpha}$ be the solution and $\alpha$-path of the uncertain differential equation

$$
d X_{t}=f\left(t, X_{t}\right) d t+g\left(t, X_{t}\right) d C_{t}
$$

respectively. Then, for any time $s>0$ and strictly increasing function $J(x)$, the supremum

$$
\sup _{0 \leq t \leq s} J\left(X_{t}\right)
$$

has an inverse uncertainty distribution

$$
\Psi_{s}^{-1}(\alpha)=\sup _{0 \leq t \leq s} J\left(X_{t}^{\alpha}\right)
$$

and the infimum

$$
\inf _{0 \leq t \leq s} J\left(X_{t}\right)
$$

has an inverse uncertainty distribution

$$
\Psi_{s}^{-1}(\alpha)=\inf _{0 \leq t \leq s} J\left(X_{t}^{\alpha}\right)
$$




\subsection{Uncertain mean-reverting currency model}

Assuming that the stock price follows a geometric Liu process, Liu [11] built the first uncertain stock model, which was applied widely

$$
\left\{\begin{array}{l}
d X_{t}=r X_{t} d t \\
d Y_{t}=e Y_{t} d t+r Y_{t} d C_{t} .
\end{array}\right.
$$

The bond price $X_{t}$ and the stock price $Y_{t}$ are determined by this model, where $r$ is the risk-free interest rate, $e$ is the stock drift, $\sigma$ is the stock diffusion and $C_{t}$ is a canonical Liu process. Then the model was derived in the international financial market by Liu et al. [20]

$$
\left\{\begin{array}{l}
d X_{t}=\mu X_{t} d t \\
d Y_{t}=\nu Y_{t} d t \\
d Z_{t}=e Z_{t} d t+\sigma Z_{t} d t .
\end{array}\right.
$$

In this model, $X_{t}$ represents the domestic currency with a domestic interest rate $\mu, Y_{t}$ is the foreign currency with a foreign interest rate $\nu, Z_{t}$ represents the exchange rate (the domestic currency price of one unit of foreign currency) at time $t$. It means that one unit of foreign currency equals to $Z_{t}$ unit of domestic currency.

It is found that the change of foreign exchange rate stays around an average level in the long term in real global financial market. Therefore mean-reverting model was proposed by Shen and Yao [21] in 2016, in which $\frac{m}{a}$ means the average level

$$
\left\{\begin{array}{l}
d X_{t}=\mu X_{t} d t \\
d Y_{t}=\nu Y_{t} d t \\
d Z_{t}=e\left(m-a Z_{t}\right) d t+\sigma Z_{t} d t
\end{array}\right.
$$

\section{Lookback call option pricing formula with the fixed strike}

\subsection{The pricing formula of lookback call option}

A lookback call option provides the holder the right to sell a certain asset at the highest price during a certain period. Assume that a buyer can buy one unit of foreign lookback option during $t \in[0, T]$ with a fixed striking price $\mathrm{K}$, which $\mathrm{T}$ is the expiration time. Then the payoff of the buyer is

$$
\left(\sup _{0 \leq t \leq T} Z_{t}-K\right)^{+},
$$

where $Z_{t}$ is the currency at time t. Considering the time value of money resulted from the bond, the present value of the payoff is

$$
\exp (-\mu T)\left(\sup _{0 \leq t \leq T} Z_{t}-K\right)^{+} .
$$

Let $f_{c}$ present the price of the call option, then the expected profit of the buyer is

$$
-f_{c}+\exp (-\mu T)\left(\sup _{0 \leq t \leq T} Z_{t}-K\right)^{+}
$$

and the expected profit of the bank is

$$
f_{c}-Z_{0} \exp (-\nu T)\left(1-\frac{K}{\sup _{0 \leq t \leq T} Z_{t}}\right)^{+} .
$$


According to the fair price principle, the buyer and the bank should gain the same expected profit

$$
E\left[-f_{c}+\exp (-\mu T)\left(\sup _{0 \leq t \leq T} Z_{t}-K\right)^{+}\right]=E\left[f_{c}-Z_{0} \exp (-\nu T)\left(1-\frac{K}{\sup _{0 \leq t \leq T} Z_{t}}\right)^{+}\right] .
$$

This the pricing formula can be presented as follows:

$$
f_{c}=\frac{1}{2} \exp (-\mu T) E\left[\left(\sup _{0 \leq t \leq T} Z_{t}-K\right)^{+}\right]+\frac{1}{2} Z_{0} \exp (-\nu T) E\left[\left(1-\frac{K}{\sup _{0 \leq t \leq T} Z_{t}}\right)^{+}\right] .
$$

Theorem 3.1. Assume the lookback call option for the stock model (3) has a strike price $K$ and an expiration time T. Then the price of the lookback call option is

$$
f_{c}=\frac{1}{2} \exp (-\mu T) \int_{0}^{1} \sup _{0 \leq t \leq T}\left(\Psi_{t}^{-1}(\alpha)-K\right)^{+} d \alpha+\frac{1}{2} Z_{0} \exp (-\nu T) \int_{0}^{1} \sup _{0 \leq t \leq T}\left(1-\frac{K}{\Psi_{t}^{-1}(\alpha)}\right)^{+} d \alpha,
$$

where

$$
\Psi_{t}^{-1}(\alpha)=Z_{0} \exp (-a t)+\frac{m+\frac{\sigma \sqrt{3}}{\pi} \ln \frac{\alpha}{1-\alpha}}{a}(1-\exp (-a t))
$$

Proof: By solving the ordinary differential equation

$$
Z_{t}^{\alpha}=\left(m-\sigma Z_{t}^{\alpha}+\sigma \Psi_{t}^{-1}(\alpha)\right) \mathrm{d} t
$$

we get the $\alpha$-path of $Z_{t}$ :

$$
\begin{aligned}
Z_{t}^{\alpha} & =Z_{0} \exp (-a t)+\frac{m+\sigma \Psi_{t}^{-1}(\alpha)}{a}(1-\exp (-a t)) \\
& =Z_{0} \exp (-a t)+\frac{m+\frac{\sigma \sqrt{3}}{\pi} \ln \frac{\alpha}{1-\alpha}}{a}(1-\exp (-a t)) .
\end{aligned}
$$

By Theorem 2.4, the foreign exchange rate $Z_{t}$ has inverse distribution function

$$
\Psi_{t}^{-1}(\alpha)=Z_{t}^{\alpha}=Z_{0} \exp (-a t)+\frac{m+\frac{\sigma \sqrt{3}}{\pi} \ln \frac{\alpha}{1-\alpha}}{a} .
$$

And since

$$
\left(\sup _{0 \leq t \leq T} Z_{t}-K\right)^{+}=\left(\sup _{0 \leq t \leq T}\left(Z_{t}-K\right)\right)^{+}=\sup _{0 \leq t \leq T}\left(Z_{t}-K\right)^{+},
$$

by Theorem 2.5, the inverse distribution of

$$
\left(\sup _{0 \leq t \leq T} Z_{t}-K\right)^{+}
$$

is

$$
\sup _{0 \leq t \leq T}\left(\Psi_{t}^{-1}(\alpha)-K\right)^{+}
$$

The inverse distribution of

$$
\left(1-\frac{K}{\sup _{0 \leq t \leq T} Z_{t}}\right)^{+}
$$

is

$$
\sup _{0 \leq t \leq T}\left(1-\frac{K}{\Psi_{t}^{-1}(\alpha)}\right)^{+} .
$$


Therefore,

$$
\begin{aligned}
f_{c} & =\frac{1}{2} \exp (-\mu T) \int_{0}^{1} \sup _{0 \leq t \leq T}\left(\Psi_{t}^{-1}(\alpha)-K\right)^{+} d \alpha+\frac{1}{2} Z_{0} \exp (-\nu T) \int_{0}^{1} \sup _{0 \leq t \leq T}\left(1-\frac{K}{\Psi_{t}^{-1}(\alpha)}\right)^{+} d \alpha \\
& =\frac{1}{2} \exp (-\mu T) \int_{0}^{1} \sup _{0 \leq t \leq T}\left(Z_{0} \exp (-a t)+\frac{m+\frac{\sigma \sqrt{3}}{\pi} \ln \frac{\alpha}{1-\alpha}}{a}(1-\exp (-a t))-K\right)^{+} d \alpha \\
& +\frac{1}{2} Z_{0} \exp (-\nu T) \int_{0}^{1} \sup _{0 \leq t \leq T}\left(1-\frac{K}{Z_{0} \exp (-a t)+\frac{m+\frac{\sigma \sqrt{3}}{\pi} \ln \frac{\alpha}{1-\alpha}}{a}(1-\exp (-a t))}\right)^{+} d \alpha .
\end{aligned}
$$

The lookback call option pricing formula is verified.

\subsection{Numerical experiment}

An algorithm is designed to calculate the lookback call option price $f_{c}$.

Step 0 Set the initial parameters as $\mathrm{K}=4, Z_{0}=5, \mu=0.05, \nu=0.04, \sigma=0.1, \mathrm{~T}=1, \mathrm{~m}=6$, $\mathrm{a}=1$.

Step 1 Set $\alpha_{i}=i / N$ and $t_{j}=j T / M, i=1,2, \ldots, N-1, j=1,2, \ldots, M$, where $\mathrm{N}$ and $\mathrm{M}$ are two large numbers.

Step 2 Set $i=0, j=0$.

Step 3 Set $i \leftarrow i+1$.

Step 4 Set $j \leftarrow j+1$.

Step 5 Set $P_{t_{0}}^{\alpha_{i}}=0$.

Step 6 Calculate the price at time $t_{j}$

$$
P_{t_{j}}^{\alpha_{i}}=\Psi_{t_{j}}^{-1}(\alpha)=Z_{0} \exp \left(-a t_{j}\right)+\frac{m+\frac{\sigma \sqrt{3}}{\pi} \ln \frac{\alpha_{i}}{1-\alpha_{i}}}{a}\left(1-\exp \left(-a t_{j}\right)\right) .
$$

If $P_{t_{j}}^{\alpha_{i}}>P_{t_{j-1}}^{\alpha_{i}}$ and $j<M$, then return to Step 4. If $P_{t_{j}}^{\alpha_{i}} \geq P_{t_{j-1}}^{\alpha_{i}}$ and $j=M$, then jump to Step 8 .

Step 7 Set $P_{t_{j}}^{\alpha_{i}} \leftarrow P_{t_{j-1}}^{\alpha_{i}}$. If $j<M$, then return to Step 4 .

Step 8 Calculate $P_{t_{j}}^{\alpha_{i}}-K, 1-\frac{K}{P_{t_{j}}^{\alpha_{i}}}$.

Step 9 Set $P^{\alpha_{i}} \leftarrow \max \left(0, P_{t_{j}}^{\alpha_{i}}-K\right), Q^{\alpha_{i}} \leftarrow \max \left(0,1-\frac{K}{P_{t_{j}}^{\alpha_{i}}}\right)$. If $i<N-1$, then return to Step 2.

Step 10 Calculate the lookback call option price

$$
f_{c} \leftarrow \frac{1}{2} \exp (-\mu T) \frac{1}{N-1} \sum_{i=1}^{N-1} P^{\alpha_{i}}+\frac{1}{2} Z_{0} \exp (-\nu T) \frac{1}{N-1} \sum_{i=1}^{N-1} Q^{\alpha_{i}} .
$$

Example 3.1. Suppose that the initial exchange rate $Z_{0}=5$, the domestic interest rate $\mu=0.05$, the foreign interest rate $\nu=0.04$, the $\log$-diffusion $\sigma=0.1$, the strike price $\mathrm{K}=4$, the expiration time $\mathrm{T}=1$, $\mathrm{m}=6, \mathrm{a}=1$. Then the price of lookback call currency option is

$$
f_{c} \approx 1.4719 \text {. }
$$

\subsection{Sensibility analysis}

Theorem 3.2. (Monotonicity of lookback call option pricing model) Suppose the lookback call option price follows the stock model (3), then $f_{c}$ has the following properties:

1. $f_{c}$ is an increasing function of $Z_{0}$;

2. $f_{c}$ is a decreasing function of $K$;

3. $f_{c}$ is a decreasing function of $\mu$;

4. $f_{c}$ is a decreasing function of $\nu$. 
Proof: 1 . Since

$$
\begin{aligned}
\frac{\mathrm{d} f_{c}}{\mathrm{~d} Z_{0}} & =\frac{1}{2} \exp (-\mu T) \int_{0}^{1} \sup _{0 \leq t \leq T} \exp (-a t) d \alpha+\frac{1}{2} \exp (-\nu T) \int_{0}^{1} \sup _{0 \leq t \leq T}\left(1-\frac{K}{\Psi_{t}^{-1}(\alpha)}\right)^{+} d \alpha \\
& +\frac{1}{2} Z_{0} \exp (-\nu T) \int_{0}^{1} \sup _{0 \leq t \leq T}\left(\frac{K \exp (-a t)}{\Psi_{t}^{-1}(\alpha)^{2}}\right) d \alpha>0 .
\end{aligned}
$$

It is obvious that $f_{c}$ is an increasing function of $Z_{0}$.

Under the assumption that other parameters except $Z_{0}$ are constants, change $Z_{0}$ from 0 to 6 with step 0.001 , we can draw the figure of lookback call option price $f_{c}$ (Fig 1 ).

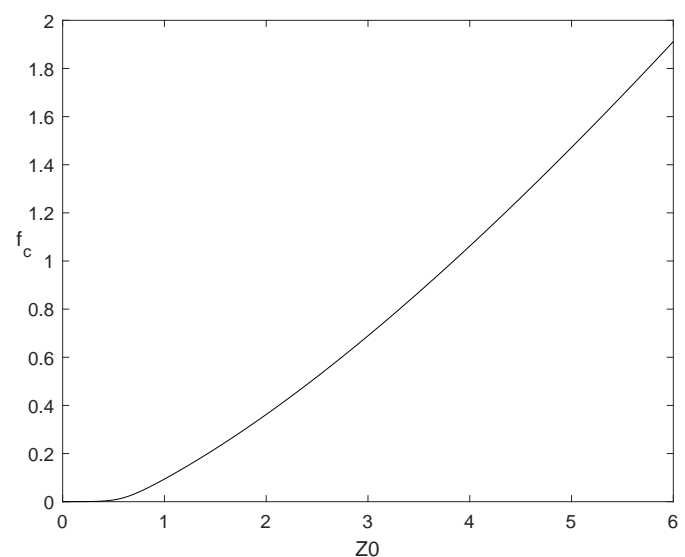

Figure 1: Price with respect to $Z_{0}$

Table 1: $f_{c}$ with the change of $Z_{0}$

\begin{tabular}{cccccc}
\hline$Z_{0}$ & 4.5 & 5 & 5.5 & 6 & 6.5 \\
\hline$f_{c}$ & 1.263 & 1.472 & 1.689 & 1.912 & 2.142 \\
\hline
\end{tabular}

2. Since

$$
\frac{\mathrm{d} f_{c}}{\mathrm{~d} K}=-\frac{1}{2} \exp (-\mu T)-\frac{1}{2} Z_{0} \exp (-\nu T) \int_{0}^{1} \sup _{0 \leq t \leq T}\left(\frac{1}{\Psi_{t}^{-1}(\alpha)}\right) d \alpha<0,
$$

$f_{c}$ is a decreasing function of $\mathrm{K}$.

Under the assumption that other parameters except $K$ are constants, change $K$ from 3 to 6 with step 0.001 , we can draw the figure of lookback call option price $f_{c}$ (Fig 2).

Table 2: $f_{c}$ with the change of $\mathrm{K}$

\begin{tabular}{cccccc}
\hline $\mathrm{K}$ & 3 & 3.5 & 4 & 4.5 & 5 \\
\hline$f_{c}$ & 2.374 & 1.923 & 1.472 & 1.021 & 0.570 \\
\hline
\end{tabular}

3. Since

$$
\frac{\mathrm{d} f_{c}}{\mathrm{~d} \mu}=-\frac{T}{2} \exp (-\mu t) \int_{0}^{1} \sup _{0 \leq t \leq T}\left(\Psi_{t}^{-1}(\alpha)-K\right)^{+} d \alpha<0,
$$

$f_{c}$ is a decreasing function of $\mu$. 


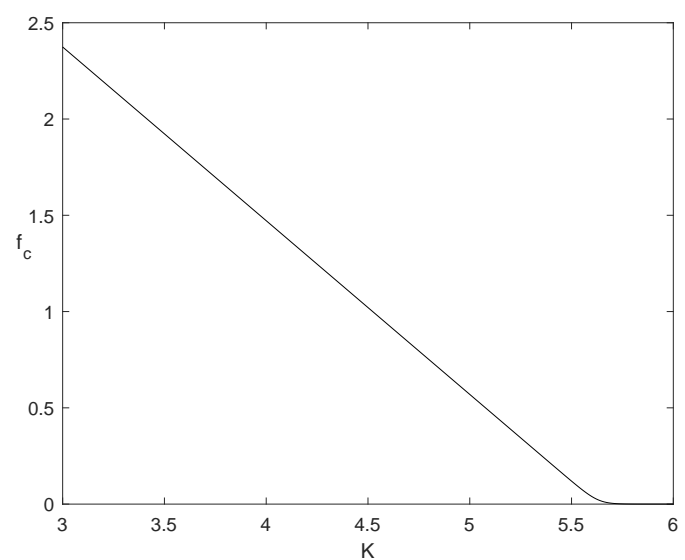

Figure 2: Price with respect to $\mathrm{K}$

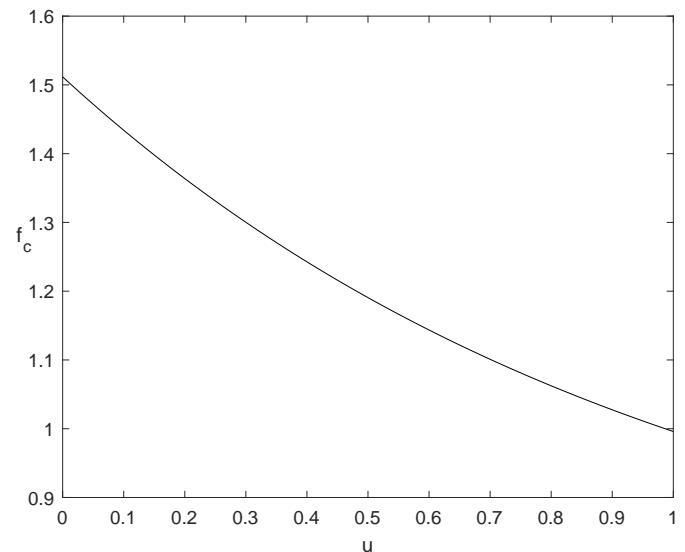

Figure 3: Price with respect to $\mu$

Table 3: $f_{c}$ with the change of $\mu$

\begin{tabular}{cccccc}
\hline$\mu$ & 0.02 & 0.05 & 0.08 & 0.11 & 0.14 \\
\hline$f_{c}$ & 1.496 & 1.472 & 1.449 & 1.427 & 1.405 \\
\hline
\end{tabular}


Under the assumption that other parameters except $\mu$ are constants, change $\mu$ from 0 to 1 with step 0.001 , we can draw the figure of lookback call option price $f_{c}$ (Fig 3$)$.

4. Since

$$
\frac{\mathrm{d} f_{c}}{\mathrm{~d} \nu}=-\frac{T}{2} \exp (-\nu t) \int_{0}^{1} \sup _{0 \leq t \leq T}\left(1-\frac{K}{\Psi_{t}^{-1}(\alpha)}\right)^{+} d \alpha<0,
$$

$f_{c}$ is a decreasing function of $\nu$.

Under the assumption that other parameters except $\nu$ are constants, change $\nu$ from 0 to 1 with step 0.001 , we can draw the figure of lookback call option price $f_{c}$ (Fig 4).

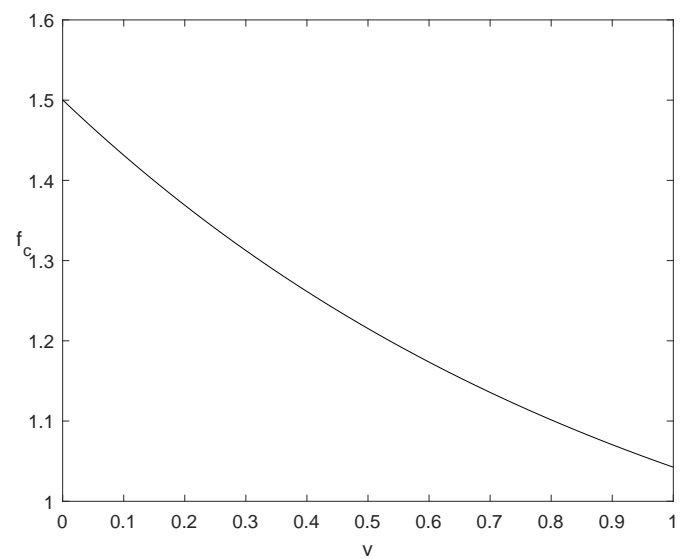

Figure 4: Price with respect to $\nu$

Table 4: $f_{c}$ with the change of $\nu$

\begin{tabular}{cccccc}
\hline$\nu$ & 0.01 & 0.04 & 0.07 & 0.10 & 0.13 \\
\hline$f_{c}$ & 1.493 & 1.472 & 1.451 & 1.431 & 1.412 \\
\hline
\end{tabular}

\section{Lookback put option pricing formula with the fixed strike}

\subsection{The pricing formula of lookback put option}

Assume that a foreign lookback put option has a fixed striking price $\mathrm{K}$ and an expiration time T. Then the payoff of a buyer is

$$
\left(K-\inf _{0 \leq t \leq T} Z_{t}\right)^{+} .
$$

Considering the time value of money resulted from the bond, the present value of the payoff is

$$
\exp (-\mu T)\left(K-\inf _{0 \leq t \leq T} Z_{t}\right)^{+}
$$

Let $f_{p}$ present the price of the lookback put option, then the expected profit of the buyer is

$$
-f_{p}+\exp (-\mu T)\left(K-\inf _{0 \leq t \leq T} Z_{t}\right)^{+}
$$

and the expected profit of the bank is

$$
f_{p}-Z_{0} \exp (-\nu T)\left(\frac{K}{\inf _{0 \leq t \leq T} Z_{t}}-1\right)^{+} .
$$


According to the fair price principle, the buyer and the bank should gain the same expected profit

$$
E\left[-f_{p}+\exp (-\mu T)\left(K-\inf _{0 \leq t \leq T} Z_{t}\right)^{+}\right]=E\left[f_{p}-Z_{0} \exp (-\nu T)\left(\frac{K}{\inf _{0 \leq t \leq T} Z_{t}}-1\right)^{+}\right] .
$$

This the pricing formula can be presented as follows:

$$
f_{p}=\frac{1}{2} \exp (-\mu T) E\left[\left(K-\inf _{0 \leq t \leq T} Z_{t}\right)^{+}\right]+\frac{1}{2} Z_{0} \exp (-\nu T) E\left[\left(\frac{K}{\inf _{0 \leq t \leq T} Z_{t}}-1\right)^{+}\right] .
$$

Theorem 4.1. Assume the lookback put option for the stock model (3) has a strike price $K$ and an expiration time T. Then the price of the lookback put option is

$$
f_{p}=\frac{1}{2} \exp (-\mu T) \int_{0}^{1} \inf _{0 \leq t \leq T}\left(K-\Psi_{t}^{-1}(\alpha)\right)^{+} d \alpha+\frac{1}{2} Z_{0} \exp (-\nu T) \int_{0}^{1} \inf _{0 \leq t \leq T}\left(\frac{K}{\Psi_{t}^{-1}(\alpha)}-1\right)^{+} d \alpha,
$$

where

$$
\Psi_{t}^{-1}(\alpha)=Z_{0} \exp (-a t)+\frac{m+\frac{\sigma \sqrt{3}}{\pi} \ln \frac{\alpha}{1-\alpha}}{a}(1-\exp (-a t))
$$

Proof: We have known that the foreign exchange rate $Z_{t}$ has inverse distribution function

$$
\Psi_{t}^{-1}(\alpha)=Z_{t}^{\alpha}=Z_{0} \exp (-a t)+\frac{m+\frac{\sigma \sqrt{3}}{\pi} \ln \frac{\alpha}{1-\alpha}}{a}
$$

Since

$$
\left(K-\inf _{0 \leq t \leq T} Z_{t}\right)^{+}=\left(\inf _{0 \leq t \leq T}\left(K-Z_{t}\right)\right)^{+}=\inf _{0 \leq t \leq T}\left(K-Z_{t}\right)^{+},
$$

the inverse distribution of

$$
\left(K-\inf _{0 \leq t \leq T} Z_{t}\right)^{+}
$$

is

$$
\inf _{0 \leq t \leq T}\left(K-\Psi_{t}^{-1}(\alpha)\right)^{+}
$$

The inverse distribution of

$$
\left(\frac{K}{0 \leq t \leq T} Z_{0}-1\right)^{+}
$$

is

$$
\inf _{0 \leq t \leq T}\left(\frac{K}{\Psi_{t}^{-1}(\alpha)}-1\right)^{+} .
$$

Therefore,

$$
\begin{aligned}
f_{p} & =\frac{1}{2} \exp (-\mu T) \int_{0}^{1} \inf _{0 \leq t \leq T}\left(K-\Psi_{t}^{-1}(\alpha)\right)^{+} d \alpha+\frac{1}{2} Z_{0} \exp (-\nu T) \int_{0}^{1} \inf _{0 \leq t \leq T}\left(\frac{K}{\Psi_{t}^{-1}(\alpha)}-1\right)^{+} d \alpha \\
& =\frac{1}{2} \exp (-\mu T) \int_{0}^{1} \inf _{0 \leq t \leq T}\left(K-Z_{0} \exp (-a t)-\frac{m+\frac{\sigma \sqrt{3}}{\pi} \ln \frac{\alpha}{1-\alpha}}{a}(1-\exp (-a t))\right)^{+} d \alpha \\
& +\frac{1}{2} Z_{0} \exp (-\nu T) \int_{0}^{1} \inf _{0 \leq t \leq T}\left(\frac{K}{Z_{0} \exp (-a t)+\frac{m+\frac{\sigma \sqrt{3}}{\pi} \ln \frac{\alpha}{1-\alpha}}{a}(1-\exp (-a t))}-1\right)^{+} d \alpha .
\end{aligned}
$$

The lookback put option pricing formula is verified. 


\subsection{Numerical experiment}

An algorithm is designed to calculate the lookback put option price $f_{p}$.

Step 0 Set the initial parameters as $\mathrm{K}=5, Z_{0}=4, \mu=0.05, \nu=0.04, \sigma=0.1, \mathrm{~T}=1, \mathrm{~m}=6, \mathrm{a}=1$.

Step 1 Set $\alpha_{i}=i / N$ and $t_{j}=j T / M, i=1,2, \ldots, N-1, j=1,2, \ldots, M$, where $\mathrm{N}$ and $\mathrm{M}$ are two large numbers.

Step 2 Set $i=0, j=0$.

Step 3 Set $i \leftarrow i+1$.

Step 4 Set $j \leftarrow j+1$.

Step 5 Set $P_{t_{0}}^{\alpha_{i}}=K$.

Step 6 Calculate the price at time $t_{j}$

$$
P_{t_{j}}^{\alpha_{i}}=\Psi_{t_{j}}^{-1}(\alpha)=Z_{0} \exp \left(-a t_{j}\right)+\frac{m+\frac{\sigma \sqrt{3}}{\pi} \ln \frac{\alpha_{i}}{1-\alpha_{i}}}{a}\left(1-\exp \left(-a t_{j}\right)\right)
$$

If $P_{t_{j}}^{\alpha_{i}} \leq P_{t_{j-1}}^{\alpha_{i}}$ and $j<M$, then return to Step 4. If $P_{t_{j}}^{\alpha_{i}} \leq P_{t_{j-1}}^{\alpha_{i}}$ and $j=M$, then jump to Step 8 .

Step 7 Set $P_{t_{j}}^{\alpha_{i}} \leftarrow P_{t_{j-1}}^{\alpha_{i}}$. If $j<M$, then return to Step 4.

Step 8 Calculate $K-P_{t_{j}}^{\alpha_{i}}, \frac{K}{P_{t_{j}}^{\alpha_{i}}}-1$.

Step 9 Set $P^{\alpha_{i}} \leftarrow \max \left(0, K-P_{t_{j}}^{\alpha_{i}}\right), Q^{\alpha_{i}} \leftarrow \max \left(0, \frac{K}{P_{t_{j}}^{\alpha_{i}}}-1\right)$. If $i<N-1$, then return to Step 2 .

Step 10 Calculate the lookback put option price

$$
f_{p} \leftarrow \frac{1}{2} \exp (-\mu T) \frac{1}{N-1} \sum_{i=1}^{N-1} P^{\alpha_{i}}+\frac{1}{2} Z_{0} \exp (-\nu T) \frac{1}{N-1} \sum_{i=1}^{N-1} Q^{\alpha_{i}} .
$$

Example 4.1. Suppose that the initial exchange rate $Z_{0}=4$, the domestic interest rate $\mu=0.05$, the foreign interest rate $\nu=0.04$, the $\log$-diffusion $\sigma=0.1$, the strike price $\mathrm{K}=5$, the expiration time $\mathrm{T}=1$, $\mathrm{m}=6, \mathrm{a}=1$. Then the price of lookback put option is

$$
f_{p} \approx 0.9595
$$

\subsection{Sensibility analysis}

Theorem 4.2. (Monotonicity of lookback put option pricing model) Suppose the lookback put currency option price follows the stock model (3), then $f_{p}$ has the following properties:

1. $f_{p}$ is an increasing function of $K$;

2. $f_{p}$ is a decreasing function of $Z_{0}$;

3. $f_{p}$ is a decreasing function of $\mu$;

4. $f_{p}$ is a decreasing function of $\nu$.

Proof: 1 . Since

$$
\frac{\mathrm{d} f_{p}}{\mathrm{~d} K}=\frac{1}{2} \exp (-\mu T)+\frac{1}{2} Z_{0} \exp (-\nu T) \int_{0}^{1} \inf _{0 \leq t \leq T}\left(\frac{1}{\Psi_{t}^{-1}(\alpha)}\right) d \alpha>0,
$$

$f_{p}$ is an increasing function of $\mathrm{K}$.

Under the assumption that other parameters except $K$ are constants, change $K$ from 3 to 6 with step 0.001 , we can draw the figure of lookback put option price $f_{p}$ (Fig 5)

2. Under the assumption that other parameters except $Z_{0}$ are constants, change $Z_{0}$ from 3 to 6 with step 0.001 , we can draw the figure of lookback put option price $f_{p}$ (Fig 6). 


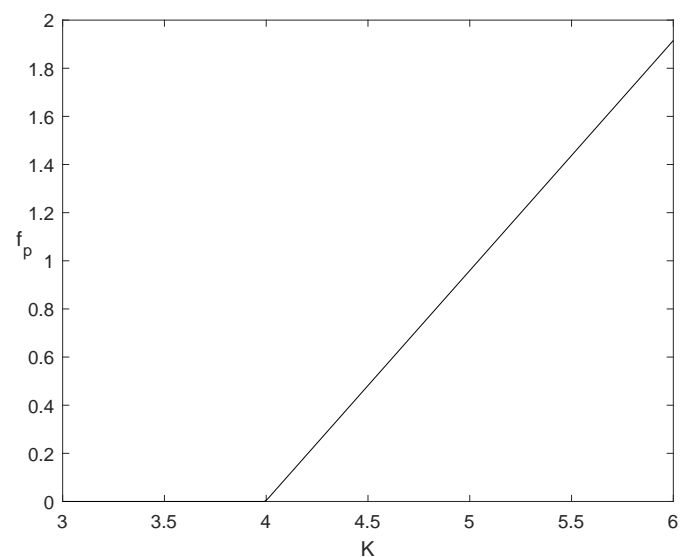

Figure 5: Price with respect to K

Table 5: $f_{p}$ with the change of $\mathrm{K}$

\begin{tabular}{cccccc}
\hline $\mathrm{K}$ & 4 & 4.5 & 5 & 5.5 & 6 \\
\hline$f_{p}$ & 0.005 & 0.482 & 0.960 & 1.437 & 1.915 \\
\hline
\end{tabular}

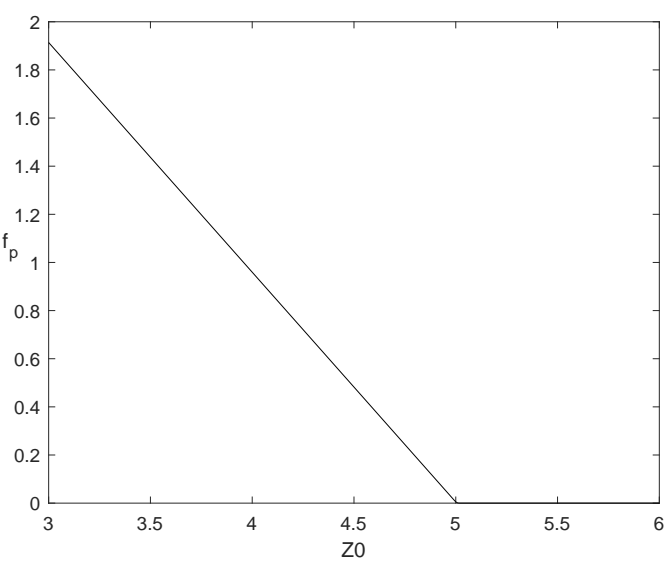

Figure 6: Price with respect to $Z_{0}$

Table $6: f_{p}$ with the change of $Z_{0}$

\begin{tabular}{cccccc}
\hline$Z_{0}$ & 3 & 3.5 & 4 & 4.5 & 5 \\
\hline$f_{p}$ & 1.914 & 1.437 & 0.960 & 0.482 & 0.005 \\
\hline
\end{tabular}


3. Since

$$
\frac{\mathrm{d} f_{p}}{\mathrm{~d} \mu}=-\frac{T}{2} \exp (-\mu t) \int_{0}^{1} \inf _{0 \leq t \leq T}\left(K-\Psi_{t}^{-1}(\alpha)\right)^{+} d \alpha<0,
$$

so $f_{p}$ is a decreasing function of $\mu$.

Under the assumption that other parameters except $\mu$ are constants, change $\mu$ from 0 to 1 with step 0.001, we can draw the figure of lookback put option price $f_{p}$ (Fig 7).

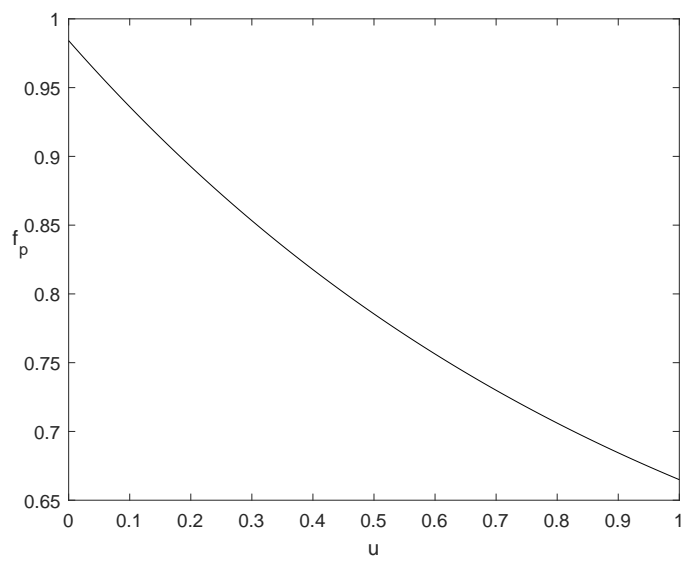

Figure 7: Price with respect to $\mu$

Table 7: $f_{p}$ with the change of $\mu$

\begin{tabular}{cccccc}
\hline$\mu$ & 0.02 & 0.05 & 0.08 & 0.11 & 0.14 \\
\hline$f_{p}$ & 0.974 & 0.960 & 0.945 & 0.932 & 0.918 \\
\hline
\end{tabular}

4. Since

$$
\frac{\mathrm{d} f_{p}}{\mathrm{~d} \nu}=-\frac{T}{2} \exp (-\nu t) \int_{0}^{1} \inf _{0 \leq t \leq T}\left(\frac{K}{\Psi_{t}^{-1}(\alpha)}-1\right)^{+} d \alpha<0,
$$

$f_{p}$ is a decreasing function of $\nu$.

Under the assumption that other parameters except $\nu$ are constants, change $\nu$ from 0 to 1 with step 0.001 , we can draw the figure of lookback put option price $f_{p}$ (Fig 8).

Table 8: $f_{p}$ with the change of $\nu$

\begin{tabular}{cccccc}
\hline$\nu$ & 0.01 & 0.04 & 0.07 & 0.10 & 0.13 \\
\hline$f_{p}$ & 0.974 & 0.960 & 0.945 & 0.932 & 0.918 \\
\hline
\end{tabular}

\section{Conclusion}

This paper mainly presented lookback options for the uncertain mean-reverting currency model. It considered lookback call currency option and lookback put option with the fixed strike, meanwhile derived their option pricing formulas. Moreover, the paper designed numerical methods to calculate the prices. Further research may consider lookback options of various uncertain stock models. 


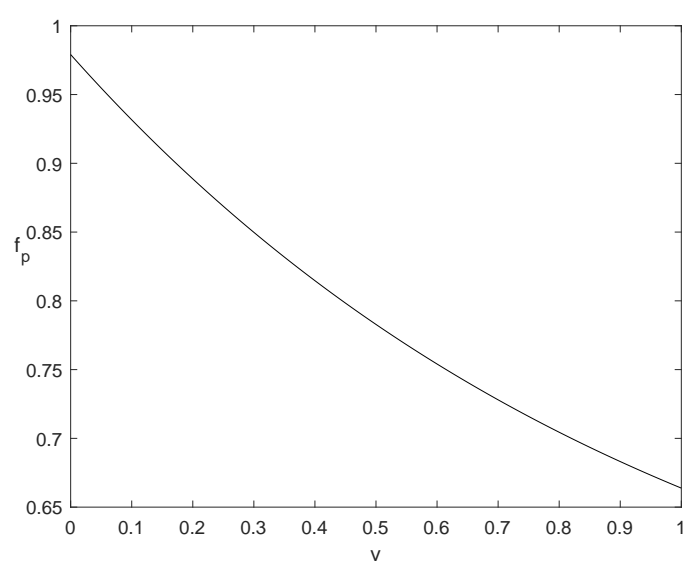

Figure 8: Price with respect to $\nu$

\section{Compliance with ethical standards}

- Funding: This research was funded by Ph.d Scientific Research Foundation of Liaocheng University (Grands No.321052022 and No.318051913) and Natural Science Foundation of Shandong Province (Grand No.ZR2020MA026). • Conflict of Interest:The authors declare that they have no competing interests.

- Research involving human participants and/or animals: This article does not contain any studies with human participants performed by any of the authors.

\section{References}

[1] L. Bachelier, Theory of speculation, Annales Scientific and Econimic 17 (1900), 21-86.

[2] K. Ito, On stochastic differential equations, American Mathematical Society 4 (1951), 1-51.

[3] F. Black and M. Scholes, The pricing of option and corporate liabilities, Journal of Political Economy 81 (1973), 637-654.

[4] R C. Merton, Theory of rational option pricing, Bell Journal of Economics and Management Science 4(1973), 141-183.

[5] B. Liu, Toward uncertain finance theory, Journal of Uncertainty Analysis and Applications 1 (2013), Article 1.

[6] B. Liu, Uncertainty Theory, 2nd ed., Springer-Verlag, Berlin, 2007.

[7] B. Liu, Theory and Practice of Uncertain Programming, 2nd ed., Springer-Verlag, Berlin, 2009.

[8] B. Liu, Uncertain logic for modeling human language, Journal of Uncertain Systems 5(1) (2011), 3-20.

[9] B. Liu, Uncertain risk analysis and uncertain reliability analysis, Journal of Uncertain Systems 4(3) (2010), 163-170.

[10] B. Liu, Fuzzy process, hybrid process and uncertain process, Journal of Uncertain Systems 2(1) (2008), 3-16.

[11] B. Liu, Some research problems in uncertainty theory, Journal of Uncertain Systems 3(1) (2009), 3-10.

[12] X.W. Chen, American option pricing formula for uncertain financial market, International Journal of Operations Research 8(2) (2011), 32-37.

[13] J.J. Sun and X.W. Chen, Asian option pricing formula for uncertain financial market, Journal of Uncertainty Analysis and Applications 3 (2015), Article 11.

[14] Z.Q. Zhang and W.Q. Liu, Geometric average Asian option pricing for uncertain financial market, Journal of Uncertain Systems 8(4) (2014), 317-320. 
[15] M.B. Goldman, H.B.Sosin and M.A. Gatto, Path dependent options: buy at the low, sell at the high, Journal of Finance 34(1979), 1111-1127.

[16] A. Conze, R. Viswanathan, Path dependent options: the case of lookback options, Journal of Finance 46(1991), 1893-1907.

[17] M. Dai, H.Y. Wong and Y.K. Kwok, Quanto lookback options, Math Finance 14:(2004), 445-467.

[18] Z.Q. Zhang, H. Ke and W.Q. Liu, Lookback options pricing for uncertain financial market, Soft Computing 23:(2019), 5537-5546

[19] Y. Gao,X.F Yang and Z.F. Fu, Lookback option pricing problem of uncertain exponential Ornstein-Uhlenbeck model,Soft Computing 22(2018), 5647-5654.

[20] Y. Liu ,X. Chen and D.A. Ralescu, Uncetain currency model and currency option pricing, Interenational Journal of Intelligent Systems 30(1)(2015), 40-51.

[21] Y.Y. Shen and K. Yao, A mean-reverting currency model in an uncertain environment, Soft Computing 20(10)(2016), 4131-4138.

[22] Y.H. Sheng and G. Shi, Mean-reverting stock model and pricingrules for Asian currency option, Journal of Intelligent and Fuzzy System 34 (2018), 4261-4268.

[23] X. Wang and Y.F. Ning, An uncertain currency model with floating interest rates, Soft Computing 21 (2017), 6739-6754.

[24] Y. Liu, W. Lio, A revision of sufficient and necessary condition of uncertainty distribution, Journal of Intelligent and Fuzzy Systems 38(4) (2020), 4845-4854.

[25] B. Liu, Uncertainty Theory: A Branch of Mathematics for Modeling Human Uncertainty, Springer-Verlag, Berlin, 2010.

[26] B. Liu, Uncertainty distribution and independence on uncertain processes, Fuzzy Optimization and Decision Making 13(3) (2014), 259-271.

[27] K. Yao and X. Chen, A numerical method for solving uncertain differential equations, Journal of Intelligent and Fuzzy Systems 25(3) (2013), 825-832.

[28] K. Yao, Extreme values and integral of solution of uncertain differential equation, Journal of Uncertainty Analysis and Applications 1 (2013), Article 2. 